\title{
Scoping review protocol: Nosocomial infections in neonatal care: a scoping review of published surveillance case definitions
}

\author{
Authors: I Heijting ${ }^{1}$, S Jansen ${ }^{4}$, M. Overbeek ${ }^{1}$, W de Boode ${ }^{1}, J_{\text {Hopman }}^{2}$, A Tossman ${ }^{3}$, V. \\ Bekker $^{4}$, M. Hogeveen ${ }^{1}$ \\ Affiliations: ${ }^{1}$ Department of Paediatrics, Division of Neonatology, Amalia Children's Hospital, \\ Radboud university medical center, Radboud Institute for Health Sciences, Nijmegen, The \\ Netherlands.
}

\begin{abstract}
2Department of Quality and Safety, Department of Medical Microbiology, Radboudumc Center for Infectious Diseases, Radboud university medical center, Nijmegen, the Netherlands
\end{abstract} 3Unit of Hygiene and Infection Control, Department of Medical Microbiology, Radboudumc Center for
Infectious Diseases, Radboud university medical center, Nijmegen, the Netherlands.

\begin{abstract}
${ }^{4}$ Department of Pediatrics, Division of Neonatology, William-Alexander Children's Hospital, Leiden University Medical Center (LUMC), Leiden, The Netherlands
\end{abstract}

Corresponding author: Hogeveen M, Department of Paediatrics, Division of Neonatology, Amalia Children's Hospital, Radboud university medical center, Radboud Institute for Health Sciences, Internal postal code 804, Geert Grooteplein Zuid 10, 6525GA, Nijmegen, the Netherlands.

Marije.Hogeveen@radboudumc.nl; ; phone: +3124-3614430.

\section{Introduction}

Nosocomial infections (NI), otherwise known as healthcare-associated infections, are defined by the World Health Organization (WHO) as an infection occurring in a patient in the process of care in a hospital or other health care facility, which was not incubating or present at the time of admission. This includes infections appearing after discharge but acquired in the health care facility as well as occupational infections among health care workers of the facility ${ }^{(1)}$. Bacteria are the most common group of pathogens that cause $\mathrm{NI}{ }^{(2)}$. $\mathrm{NI}$ continue to be a serious public health issue worldwide even though it is estimated that approximately one third of these infections could be prevented by effective infection prevention programs ${ }^{(3)}$. Critically-ill infants admitted to the neonatal intensive care unit (NICU) in particular, are at an increased risk of neonatal nosocomial infections (NNI) due to impaired host-defense mechanisms, concomitant medical conditions, prolonged hospitalizations and frequent and long-lasting use of invasive medical devices ${ }^{(4)}$.

$\mathrm{NNI}$ are associated with significant morbidity and mortality. The type of morbidity is determined by the type of NNI. For example, an episode of hospital-acquired meningitis may lead to visual impairment, hearing loss, seizure disorder, mental retardation, cerebral palsy and learning disabilities ${ }^{(5,6)}$ while an episode of ventilator-associated pneumonia may lead to chronic lung diseases ${ }^{(7)}$. Mortality rates are reported from $13 \%$ to $50 \%$ in neonates who experience one or more episodes of NNI, specifically those with a bloodstream infection or meningitis ${ }^{(8)}$. $\mathrm{NNI}$ impact the healthcare system by increasing the cost of healthcare, the length of hospital stay and the use of medical resources.

There is a wide variation in the reported incidence rate of NNI. For example, in the United States NNI incidence rates vary from $6 \%$ to more than $40 \%$, but internationally incidence rates have been reported up to $69 \%{ }^{(9-14)}$. This wide variation in incidence rates may be partially explained by the lack of standardized NNI surveillance case definitions ${ }^{(15)}$ and the population at risk. Specifically, for NNI various definitions are used for differentiation between 
early onset (perinatal acquired) and late onset (nosocomial acquired) infection. For example, criteria established by the US National Healthcare Surveillance Network (NHSN) in partnership with the Centers for Disease Control and Prevention(CDC) include 48 hours as the cutoff time ${ }^{(16)}$ while the National Institute of Child Health and Human Development (NICHD) Neonatal Research Network applies a period of three days ${ }^{(10)}$. On top of that, neonatal textbooks typically use five to seven days ${ }^{(8,17)}$. Moreover, there are several different elements available for defining NNI case definitions such as specific diagnostic and clinical factors. Most reports use a positive culture, whether blood, urine or spinal fluid ${ }^{(9-14)}$. However, some other studies also include neonates with only clinical symptoms of infection who had negative cultures ${ }^{(18)}$. Furthermore, there are different ways to report NNI. For example, neonates with a central venous line and a positive blood culture are reported either as the number of positive blood cultures per catheter days or as a percentage of the population ${ }^{(19)}$. Evidently, the use of different surveillance case definitions may hamper reliable comparisons of $\mathrm{NNI}$ incidence rates on both a national and international level.

The surveillance of NNI has been shown to be an indispensable tool in infection control. It is used for defining patients who are at risk, to facilitate preventive measures and quality improvement and to detect best practice management and prevention strategies, with the ultimate aim to improve neonatal outcomes ${ }^{(20)}$. The methods that are used in published literature consist of manual, semi-automatic and automatic approaches. Through manual surveillance, patient charts are reviewed by hand while applying a case definition to detect NNI. With semiautomatic surveillance, selected cases are manually chart reviewed and 'risk cases' are selected through an electronic algorithm. Via automatic surveillance, there is no manual evaluation and a standardized definition is applied by using available electronic data ${ }^{(21)}$. The surveillance method used affects the degree in which certain surveillance criteria can be applied. For example, clinical criteria are suitable for manual surveillance but less appropriate for automated surveillance. Therefore, it is important that the surveillance criteria are adapted to the chosen surveillance method.

$\mathrm{NNI}$ surveillance data are often incomparable due to differences in defining, identifying and reporting $\mathrm{NNI}^{(19)}$. To generate reliable and valid surveillance data, standardized surveillance using uniform and unambiguously defined surveillance case definitions for the different NNI subtypes is required. As a first step towards standardized NNI surveillance, we aim to provide an overview of the available NNI surveillance case definitions in current literature and the different surveillance methods (manual, semiautomatic, automatic) used. Subsequently, we will suggest uniform NNI surveillance case definitions per subtype with the most frequently included elements.

\section{Objectives and research question \\ Objectives:}

1. To systematically review published surveillance case definitions for NNI in low, middle and high resource settings in the past two decades. This includes providing an overview of the surveillance case definitions of NNI subtypes, including:
a. Bloodstream infection (BSI)
b. Central-line associated bloodstream infection (CLABSI)
c. Ventilator-associated pneumonia (VAP)
d. Hospital-acquired pneumonia, not-ventilator associated (HAP)
e. Urinary tract infection (UTI)
f. Catheter-associated Urinary Tract Infections (CAUTI)
g. Hospital-acquired meningitis
h. Surgical site infections (SSI)

2. To evaluate published active surveillance methods for the detection of NNI. 


\section{Research questions:}

1. Which case definitions have been used for surveillance of neonatal nosocomial infections in low, middle and high resource settings in the past two decades?

2. Which surveillance methods have been used for the detection of $\mathrm{NNI}$ : manual/semi-automatic/automatic?

\section{Methods}

The review protocol has been written conform the guidelines of the Joanna Briggs Institute (JBI) manual(22) and will be registered within the Open Science Framework (OSF) database. The proposed scoping review will be performed according to the PRISMA-ScR checklist and the PRISMA flowchart for scoping reviews ${ }^{(23,24)}$. Research objectives, inclusion and exclusion criteria and methodological strategies are defined before study commencement.

\section{Inclusion and exclusion criteria}

The following inclusion and exclusion criteria will be used.

\section{Inclusion criteria:}

- Articles describing neonates born at all gestational ages who are admitted to neonatal hospital care.

- Articles have to provide the surveillance case definitions for neonatal nosocomial infections.

- Only studies published in English, German or Dutch.

- Only studies that are published between 2000 and 2020.

\section{Exclusion criteria:}

- Studies only reporting on infant populations after discharge of neonatal hospital care.

- Studies only reporting infections that were present at the time of admission at the neonatal care.

- Studies only reporting infections caused by viruses and fungi/yeasts.

- Studies only reporting infections caused by group B streptococcus

- Conference abstracts and research meetings, since these generally lack a clear description of definitions.

\section{Search strategy for identification of studies}

A comprehensive list of search terms will be used for each database including key words such as: neonates, nosocomial infection and epidemiological monitoring as well as spelling alternatives and related terms. This list is made with the help of an experienced librarian and conform the Peer Review of Electronic Search Strategies (PRESS) 2015 Evidence-Based Checklist ${ }^{(25)}$.

\section{Search strategy:}

"Cross infection"[MeSH] OR "Catheter-Related Infections"[Mesh] OR "Pneumonia, ventilator associated" [MeSH] OR "Healthcare-Associated Pneumonia" [MeSH] OR "Urinary Tract Infections"[Mesh] OR "Neonatal Sepsis" [Mesh] OR "Meningitis" [MeSH] OR "Surgical Wound Infection" [MeSH] OR Cross infection*[TIAB] OR Urinary tract infection*[TIAB] OR Meningitis [TIAB] OR Pachymeningitis [TIAB] OR Nosocomial infection*[TIAB] OR Bloodstream infection* [TIAB] OR Bacterial infection*[TIAB] OR Sepsis [TIAB] OR (Ventilator [TIAB] AND complication*[TIAB]) OR Pneumonia [TIAB] OR ((Health care [TIAB] OR healthcare [TIAB] OR hospital [TIAB] OR ventilator [TIAB] OR central line [TIAB] OR catheter [TIAB] OR surgical [TIAB] OR post-operative [TIAB]) AND infection*[TIAB]) OR HAP [TIAB] OR HABSI [TIAB] OR HABSIs [TIAB] OR LOS [TIAB] OR CLABSI [TIAB] OR CLABSIs 
[TIAB] OR CABSI [TIAB] OR CRBSI [TIAB] OR BSI [TIAB] OR VAP [TIAB] OR UTI [TIAB] OR CAUTI [TIAB] OR CAUTIS [TIAB] OR SSI [TIAB] OR SSIS [TIAB]

AND

"Intensive Care Units, neonatal"[MeSH] OR "Infant, newborn"[MeSH] OR NICU*[tiab] OR Infant ${ }^{\star}[$ TIAB] OR Newborn*[TIAB] OR Neonat* [TIAB] OR Baby [TIAB] OR Babies [TIAB]

AND

"Epidemiological monitoring"[MeSH] OR "Sentinel Surveillance"[Mesh] OR "epidemiology"[MeSH] OR "benchmarking"[MeSH] OR "infection control"[MeSH] OR "Cross infection/prevention and control"[MeSH] OR "Infections/epidemiology"[Mesh] OR Epidemiological monitoring [TIAB] OR surveillance [TIAB] OR benchmarking [TIAB] OR infection control [TIAB] OR epidemiology [TIAB]

This search strategy is suitable for PubMed(Medline) and will be adjusted for Embase, Cochrane library and Web of Science. Gray literature and unpublished studies will not be searched given the broad topic.

\section{Source of evidence selection}

The search strategy will be applied in PubMed (Medline), EMBASE, Cochrane collaboration and Web of Science. All the results will be imported to EndnoteX9 and duplicates will be excluded. The eventually articles, without the duplicates, will be imported into Rayyan. Using this program, titles and abstracts will be independently reviewed against the selection criteria and will be marked as "clearly not eligible" and "possibly eligible" by two reviewers. Discrepancies will be resolved by discussion and consensus with a third party. Studies classified as "clearly not eligible" will be excluded and all studies classified as "possibly eligible" will be moved to the full-text review. Two reviewers will again independently review the full-text articles against the selection criteria. References of the included full-text articles will be hand searched. Again discrepancies will be resolved by discussion and consensus with a third party. Through different labels in Rayyan the reason for exclusion will be maintained for every excluded article.

\section{Data extraction and synthesis}

Data extraction will be performed on included articles using a pilot tested data file. Basic information such as title, first author, country, and year of publication will be collected. The country of origin of the studies are divided into low, middle or high income countries according the World Bank country income classifications ${ }^{(26)}$. The surveillance case definitions will be presented narrative and in the form of multiple tables. These tables will give an overview of the included elements in the surveillance case definitions per NNI subtype.

\section{Discussion}

The strength of a scoping review is the use of a transparent and reproducible procedure. Through publishing this scoping review protocol in OSF, we strengthen the clarity and transparency of the research. Any alterations to this protocol will be reported in the final published scoping review.

This scoping review focuses on the lack of standardized NNI surveillance case definitions on national and international level. It aims to provide a broad overview of the surveillance case definitions used to define NNI that can be found in the published literature. This overview will include the most frequent elements used in surveillance NNI case definitions for every NNI 
subtype. This study will provide a starting point for the development of standardized surveillance case definitions. These standardized surveillance case definitions are needed to justify, monitor and refine best practice management and infection prevention strategies. Ultimately, this will lead to reduction of $\mathrm{NNI}$ incidence rates which will potentially result in shorter hospitalization, less morbidity and lower hospital costs.

\section{Limitations}

Our planned review comes with some limitations. Although we search different databases and hand search references we will miss some studies. First because we do not search gray literature and secondly not all full-text will be available so we will have to reach out to authors and they may not respond and we might miss some relevant work. We may not be able to make a conclusion including a surveillance case definition with the most frequent elements used for every subtype if there are too few published studies which contain a surveillance case definition.

\section{Reference list}

1. Landelle C, Pittet D. Oxford Textbook of Critical Care. Definition, epidemiology, and general management of nosocomial infection: Oxford University Press; 2016.

2. Kollef MH, Torres A, Shorr AF, Martin-Loeches I, Micek ST. Nosocomial Infection. Crit Care Med. 2021;49(2):169-87.

3. Haley RW, Culver DH, White JW, Morgan WM, Emori TG, Munn VP, et al. The efficacy of infection surveillance and control programs in preventing nosocomial infections in US hospitals. Am J Epidemiol. 1985;121(2):182-205.

4. Advani SD, Murray TS, Murdzek CM, Aniskiewicz MJ, Bizzarro MJ. Shifting focus toward healthcare-associated bloodstream infections: The need for neonatal intensive care unit-specific NHSN definitions. Infect Control Hosp Epidemiol. 2020;41(2):181-6.

5. Klinger G, Chin CN, Beyene J, Perlman M. Predicting the outcome of neonatal bacterial meningitis. Pediatrics. 2000;106(3):477-82.

6. Bedford H, de Louvois J, Halket S, Peckham C, Hurley R, Harvey D. Meningitis in infancy in England and Wales: follow up at age 5 years. Bmj. 2001;323(7312):533-6.

7. Davis JM AS. Bronchopulmonary dysplasia. 2nd ed. Philadelphia: Spitzer AR, ed. Intensive Care of the Fetus and Neonate.; 2005.

8. Gomella TL CM, Eyal FG, Zenk KE. Infectious diseases. 4th ed. New York: In: Gomella TL, Cunningham MD, Eyal FG, Zenk KE, eds. Neonatology: Management, Procedures, On-Call Problems, Diseases and Drugs; 2004.

9. Zafar N, Wallace CM, Kieffer P, Schroeder P, Schootman M, Hamvas A. Improving survival of vulnerable infants increases neonatal intensive care unit nosocomial infection rate. Arch Pediatr Adolesc Med. 2001;155(10):1098-104.

10. Stoll BJ, Hansen N, Fanaroff AA, Wright LL, Carlo WA, Ehrenkranz RA, et al. Lateonset sepsis in very low birth weight neonates: the experience of the NICHD Neonatal Research Network. Pediatrics. 2002;110(2 Pt 1):285-91.

11. Clark R, Powers R, White R, Bloom B, Sanchez P, Benjamin DK, Jr. Nosocomial infection in the NICU: a medical complication or unavoidable problem? J Perinatol. 2004;24(6):382-8.

12. Sohn $A H$, Garrett DO, Sinkowitz-Cochran RL, Grohskopf LA, Levine GL, Stover BH, et al. Prevalence of nosocomial infections in neonatal intensive care unit patients: Results from the first national point-prevalence survey. J Pediatr. 2001;139(6):821-7.

13. Schelonka RL, Scruggs S, Nichols K, Dimmitt RA, Carlo WA. Sustained reductions in neonatal nosocomial infection rates following a comprehensive infection control intervention. J Perinatol. 2006;26(3):176-9. 
14. Hwang JH, Choi CW, Chang YS, Choe YH, Park WS, Shin SM, et al. The efficacy of clinical strategies to reduce nosocomial sepsis in extremely low birth weight infants. J Korean Med Sci. 2005;20(2):177-81.

15. van der Zwet WC, Kaiser AM, van Elburg RM, Berkhof J, Fetter WP, Parlevliet GA, et al. Nosocomial infections in a Dutch neonatal intensive care unit: surveillance study with definitions for infection specifically adapted for neonates. J Hosp Infect. 2005;61(4):300-11. 16. Heath JA, Zerr DM. Infections Acquired in the Nursery: Epidemiology and Control. Infectious Diseases of the Fetus and Newborn Infant. 2006:1179-205.

17. Harris MC CJ. Prevention and treatment of neonatal sepsis. 2nd ed. Philadelphia: In: Spitzer AR, ed. Intensive Care of the Fetus \& Neonate; 2005.

18. Higuera F, Rangel-Frausto MS, Rosenthal VD, Soto JM, Castañon J, Franco G, et al. Attributable cost and length of stay for patients with central venous catheter-associated bloodstream infection in Mexico City intensive care units: a prospective, matched analysis. Infect Control Hosp Epidemiol. 2007;28(1):31-5.

19. Newby J. Nosocomial Infection in Neonates: Inevitable or Preventable? The Journal of Perinatal \& Neonatal Nursing. 2008;22(3):221-7.

20. Vandenbroucke-Grauls C, Schultsz C. Surveillance in infection control: are we making progress? Curr Opin Infect Dis. 2002;15(4):415-9.

21. van Mourik MSM, Perencevich EN, Gastmeier P, Bonten MJM. Designing Surveillance of Healthcare-Associated Infections in the Era of Automation and Reporting Mandates. Clinical Infectious Diseases. 2017;66(6):970-6.

22. 11.2 Development of a scoping review protocol: Joanna Briggs Institute 2020 [Available from:

https://wiki.jbi.global/display/MANUAL/11.2+Development+of+a+scoping+review+protocol. 23. Rosie Hanneke YA, Lisa Lieberman, Leah Christina Neubauer, Michael Fagen. The Scoping Review Method: Mapping the Literature in "Structural Change" Public Health Interventions. SAGE Publications Ltd. 2016.

24. Tricco AC, Lillie E, Zarin W, O'Brien KK, Colquhoun H, Levac D, et al. PRISMA Extension for Scoping Reviews (PRISMA-ScR): Checklist and Explanation. Ann Intern Med. 2018;169(7):467-73.

25. McGowan J, Sampson M, Salzwedel DM, Cogo E, Foerster V, Lefebvre C. PRESS Peer Review of Electronic Search Strategies: 2015 Guideline Statement. Journal of Clinical Epidemiology. 2016;75:40-6.

26. Team WBD. New country classifications by income level: 2019-2020: World bank blogs; 2019 [Available from: https://blogs.worldbank.org/opendata/new-countryclassifications-income-level-2019-2020. 